\title{
CLEFMA Activates the Extrinsic and Intrinsic Apoptotic Processes through JNK1/2 and p38 Pathways in Human Osteosarcoma Cells
}

\author{
Jia-Sin Yang ${ }^{1,2}$, Renn-Chia Lin ${ }^{2,3,4,5}$, Yi-Hsien Hsieh ${ }^{6}$, Heng-Hsiung $\mathrm{Wu}^{7,8,9}{ }^{\circ}$, \\ Geng-Chung Li ${ }^{6}$, Ya-Chiu Lin ${ }^{2}$, Shun-Fa Yang ${ }^{1,2, * 10}$ and Ko-Hsiu Lu ${ }^{3,4, *}$ \\ 1 Department of Medical Research, Chung Shan Medical University Hospital, Taichung 402, Taiwan \\ 2 Institute of Medicine, Chung Shan Medical University, Taichung 402, Taiwan \\ Department of Orthopedics, Chung Shan Medical University Hospital, Taichung 402, Taiwan \\ School of Medicine, Chung Shan Medical University, Taichung 402, Taiwan \\ 5 Division of Hyperbaric Oxygen Therapy and Wound Medicine, Chung Shan Medical University Hospital, \\ Taichung 402, Taiwan \\ 6 Institute of Biochemistry, Microbiology and Immunology, Chung Shan Medical University, \\ Taichung 402, Taiwan \\ 7 Graduate Institute of Biomedical Science, China Medical University, Taichung 404, Taiwan \\ 8 Research Center of Tumor Medical Science, China Medical University, Taichung 404, Taiwan \\ 9 Center for Molecular Medicine, China Medical University Hospital, Taichung 404, Taiwan \\ * Correspondence: ysf@csmu.edu.tw (S.-F.Y.); cshy307@csh.org.tw (K.-H.L.); \\ Tel.: +886-4-24739595-34253 (S.-F.Y.)
}

Academic Editor: Roberto Fabiani

Received: 26 July 2019; Accepted: 5 September 2019; Published: 9 September 2019

check for updates

\begin{abstract}
Due to the poor prognosis of metastatic osteosarcoma, chemotherapy is usually employed in the adjuvant situation to improve the prognosis and the chances of long-term survival. 4-[3,5-Bis(2-chlorobenzylidene)-4-oxo-piperidine-1-yl]-4-oxo-2-butenoic acid (CLEFMA) is a synthetic analog of curcumin and possesses anti-inflammatory and anticancer properties. To further obtain information regarding the apoptotic pathway induced by CLEFMA in osteosarcoma cells, microculture tetrazolium assay, annexin V-FITC/PI apoptosis staining assay, human apoptosis array, and Western blotting were employed. CLEFMA dose-dependently decreased the cell viabilities of human osteosarcoma U2OS and HOS cells and significantly induced apoptosis in human osteosarcoma cells. In addition to the effector caspase 3, CLEFMA significantly activated both extrinsic caspase 8 and intrinsic caspase 9 initiators. Moreover, CLEFMA increased the phosphorylation of extracellular signal-regulated protein kinases (ERK)1/2, c-Jun N-terminal kinases (JNK) $1 / 2$ and p38. Using inhibitors of JNK (JNK-in-8) and p38 (SB203580), CLEFMA's increases of cleaved caspases 3, 8, and 9 could be expectedly suppressed, but they could not be affected by co-treatment with the ERK inhibitor (U0126). Conclusively, CLEFMA activates both extrinsic and intrinsic apoptotic pathways in human osteosarcoma cells through JNK and p38 signaling. These findings contribute to a better understanding of the mechanisms responsible for CLEFMA's apoptotic effects on human osteosarcoma cells.
\end{abstract}

Keywords: apoptosis; CLEFMA; JNK; osteosarcoma; p38

\section{Introduction}

Osteosarcoma, the most common histological form of primary bone cancer, is most prevalent in teenagers and young adults $[1,2]$. Surgical en bloc resection of the cancer to achieve a complete radical excision has been the treatment of choice for osteosarcoma [2], but its prognosis is poor because of its highly metastatic potential. To decrease its high treatment failure and mortality rates, 
the combination of surgery and chemotherapy for osteosarcoma has increased long-term survival chances to approximately $68 \%$ through limb-sparing surgeries based on radiological staging, surgical techniques, and new chemotherapy protocols [2,3]. Nevertheless, potent metastatic lung diseases are still responsible for one of the most lethal pediatric malignancies to date. Because of this, novel agents that target particular intracellular signaling pathways related to the distinctive properties of osteosarcoma cells need to be developed.

Apoptosis, or programmed cell death, a key regulator of physiological growth control and regulation of tissue homeostasis, is characterized by typical morphological and biochemical hallmarks, including cell shrinkage, nuclear DNA fragmentation and membrane blebbing [4]. Multiple stress-inducible molecules, such as mitogen-activated protein kinase (MAPK)/extracellular signal-regulated protein kinase (ERK), c-Jun N-terminal kinase (JNK), and nuclear factor kappa B (NF-kB), have been implied in transmitting the apoptotic pathway $[5,6]$. To undergo apoptosis, the activation of important initiator and effector caspases would be initiated through the activation of the extrinsic (receptor) pathway or the stimulation of the intrinsic (mitochondria) pathway [7-9]. Currently, most anticancer strategies in clinical oncology focus on triggering apoptosis in cancer cells. On the contrary, failure to undergo apoptosis may result in treatment resistance. Thereby, understanding the molecular events that regulate apoptosis in response to chemotherapy provides novel opportunities to develop molecular-targeted therapy through the intrinsic and/or extrinsic pathways for osteosarcoma, which is very difficult to cure.

Curcumin (diferuloylmethane), a bright yellow chemical produced by Curcuma longa plants, has been shown to exhibit antioxidant, anti-inflammatory, antibacterial, antiviral, antifungal, and anticancer activities through the modulation of multiple cell signaling pathways [10]. The potent cytotoxic activity of curcumin on osteosarcoma cells has been reported to be mediated by the induction of multiple apoptotic processes [11-15]. However, even though curcumin is safe at high doses (12 g/day) for humans, many reasons, such as its poor absorption, rapid metabolism, and rapid systemic elimination, contribute to the low plasma and tissue levels of curcumin [16]. To improve the poor bioavailability of curcumin, numerous approaches have been undertaken, including the use of adjuvants and structural analogues of curcumin (e.g., EF24 [3,5-bis(2-fluorobenzylidene) piperidin-4-one]).

4-[3,5-Bis(2-chlorobenzylidene)-4-oxo-piperidine-1-yl]-4-oxo-2-butenoic acid (CLEFMA) is a synthetic analog of EF 24 and possesses anti-inflammatory and anticancer properties [17,18]. Using a reverse-phase high-performance liquid chromatography (HPLC) method to analyze the stability of the new drug, CLEFMA has been validated as a potential active anticancer drug-product [19]. In fact, various signaling pathways involved in diverse antitumor properties all depend on different specific tumor types and cell lines. Despite the absence of apoptosis, the curcuminoid CLEFMA has an anti-proliferative activity to induce autophagic cell death via oxidative stress in human lung adenocarcinoma H441 cells, offering an alternative mode of cell death in apoptosis-resistant cancers [17]. Moreover, CLEFMA-induced cell death and tumor growth suppression has been reported to be associated with the cleavage of caspases 3/9 and NF-kB-regulated anti-inflammatory and anti-metastatic effects [20]. As a potent diphenyldihaloketone analogue, CLEFMA has been developed over the past years as an anticancer agent [17]; nonetheless, the effect of CLEFMA on human osteosarcoma cell death remains unclear. Thus, we investigated whether CLEFMA affects the apoptosis of osteosarcoma and attempted to define its underlying mechanisms.

\section{Results}

\subsection{Cytotoxicity of CLEFMA in Osteosarcoma U2OS and HOS Cells}

To assess the cytotoxicity of CLEFMA on osteosarcoma U2OS and HOS cells, the [3-(4,5dimethylthiazol-2-yl)-2,5-diphenyltetrazolium bromide] (MTT) assay was utilized. After $24 \mathrm{~h}$ of treatment, the viabilities of U2OS and HOS cells in the presence of concentrations of 5, 10, 20, 40 and $80 \mu \mathrm{M}$ of CLEFMA were significantly different to that of the controls $(0 \mu \mathrm{M})$ (Figure $1 \mathrm{~A}, \mathrm{~B})$, and both of the relationships were dose-dependent $(p<0.001$ and $p<0.001)$. Moreover, a $24 \mathrm{~h}$ treatment with 
$20 \mu \mathrm{M}$ of CLEFMA showed about a 50\% reduction, while a $24 \mathrm{~h}$ treatment with $80 \mu \mathrm{M}$ of CLEFMA decreased the cell viability of U2OS cells by about $90 \%$. In HOS cells, there were reductions of about $70 \%$ in $20 \mu \mathrm{M}$ and about $90 \%$ in $80 \mu \mathrm{M}$ of CLEFMA.
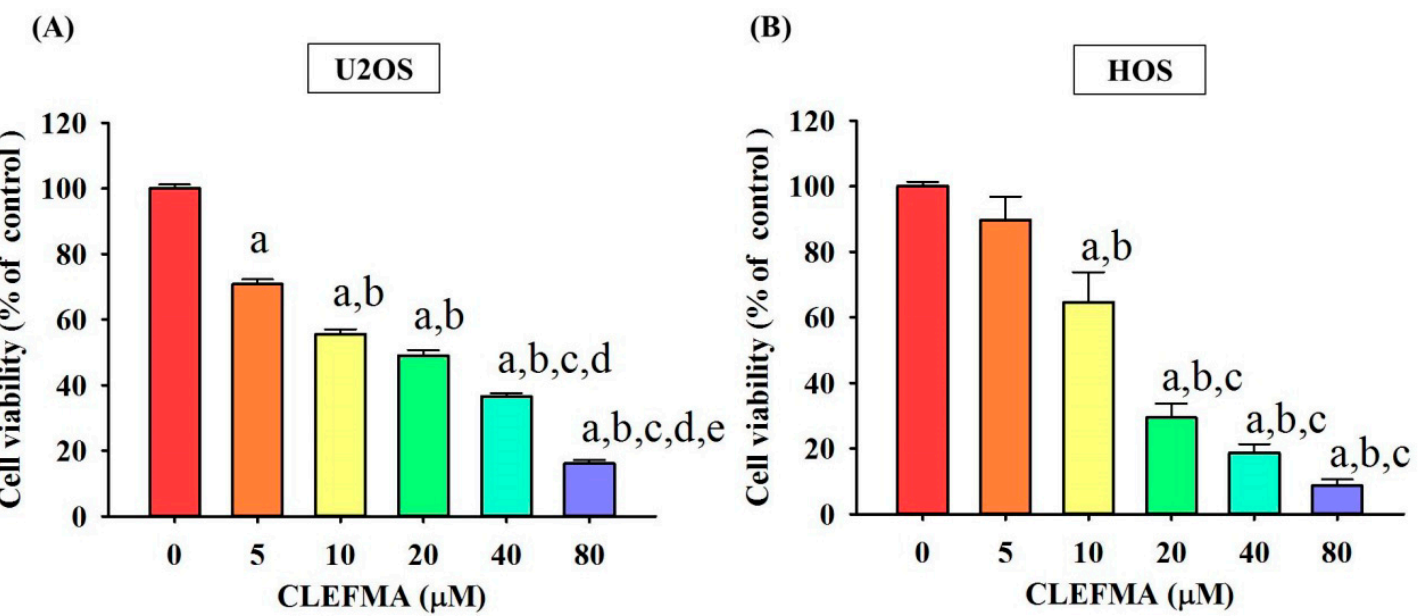

Figure 1. Effects of 4-[3,5-Bis(2-chlorobenzylidene)-4-oxo-piperidine-1-yl]-4-oxo-2-butenoic acid (CLEFMA) on the cell viability of U2OS and HOS cells. Using an [3-(4,5-dimethylthiazol-2-yl)-2,5diphenyltetrazolium bromide] (MTT) assay, the viability of U2OS and HOS cells treated with CLEFMA $(5,10,20,40$ and $80 \mu \mathrm{M})$ for $24 \mathrm{~h}$ was detected, and the effects are illustrated after quantitative analysis. Results are shown as mean \pm S.D. (A) $n \geq 4$. ANOVA analysis with Scheffe's posteriori comparison was used. $\mathrm{F}=386.619, p<0.001$. (B) $n \geq 4$. ANOVA analysis with Turkey's posteriori comparison was used. $\mathrm{F}=53.288, p<0.001$. a: Significantly different, $p<0.05$, when compared to control. b: Significantly different, $p<0.05$, when compared to $5 \mu \mathrm{M}$. c: Significantly different, $p<0.05$, when compared to $10 \mu \mathrm{M}$. d: Significantly different, $p<0.05$, when compared to $20 \mu$ M. e: Significantly different, $p<0.05$, when compared to $40 \mu \mathrm{M}$.

\subsection{CLEFMA Induces the Apoptosis of U2OS and HOS Cells}

To further examine the mechanism of CLEFMA inhibition of osteosarcoma cell proliferation, the annexin V-FITC/PI apoptosis assay was performed to test the viability of U2OS and HOS cells after a treatment of 5,10 , and $20 \mu \mathrm{M}$ of CLEFMA for $24 \mathrm{~h}$. The results revealed that the percentage of apoptotic cells was significantly increased in a dose-dependent manner (Figure 2A,B). These findings suggest that CLEFMA induced the apoptosis of osteosarcoma cells.

\subsection{CLEFMA Increases the Expression of Cleaved Caspase 3 in U2OS Cells}

To identify the underlying mechanism of apoptosis induced by CLEFMA in U2OS cells, we first employed the human apoptosis array to determine apoptosis-related proteins in U2OS cells. Consequently, obvious increases in the expression of cleaved caspase 3, HIF- $1 \alpha, \mathrm{HO}-1, \mathrm{HSP} 60$, survivin and clusterin in U2OS cells were observed after treatment with $20 \mu \mathrm{M}$ CLEFMA for $24 \mathrm{~h}$. (Figure 3) Among them, the protein that increased the most in quantity was cleaved caspase 3 , which was seven-fold that of the original, suggesting that the effector caspase 3 is responsible for the actual dismantling of the U2OS cell. 
(A)
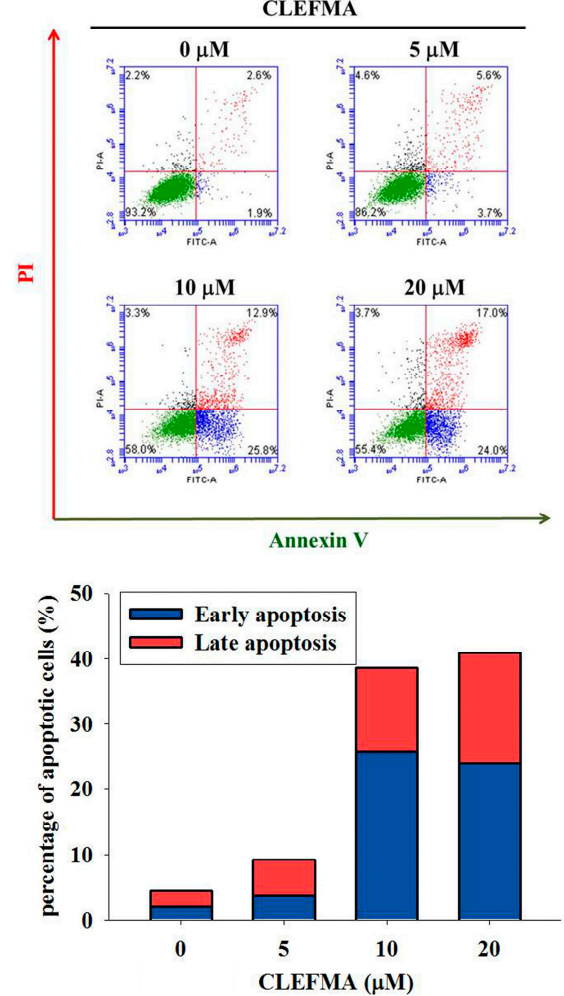

(B)
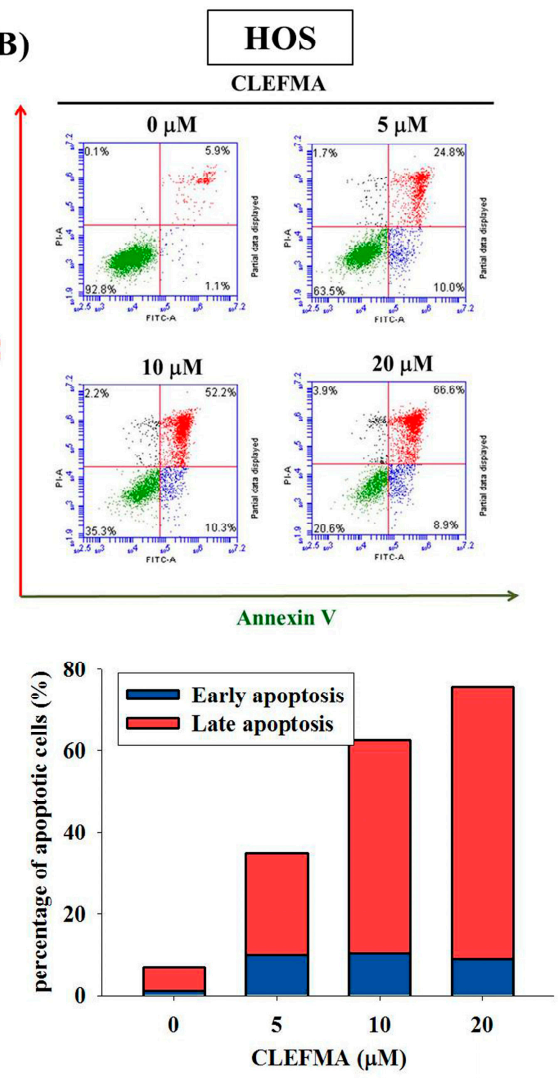

Figure 2. Effects of CLEFMA on the apoptosis of U2OS and HOS cells. (A) U2OS and (B) HOS cells were treated with CLEFMA $(5,10$ and $20 \mu \mathrm{M})$ for $24 \mathrm{~h}$ and then subjected to flow cytometry after annexin V-FITC/PI staining. Cells that were considered viable were FITC annexin V and PI negative, cells that were in early apoptosis were FITC annexin V positive and PI negative, and cells that were in late apoptosis or already dead were both FITC annexin V and PI positive. Thus, the quantitative analysis of early apoptosis and late apoptosis was summarized to differentiate apoptosis from necrosis.

(A)

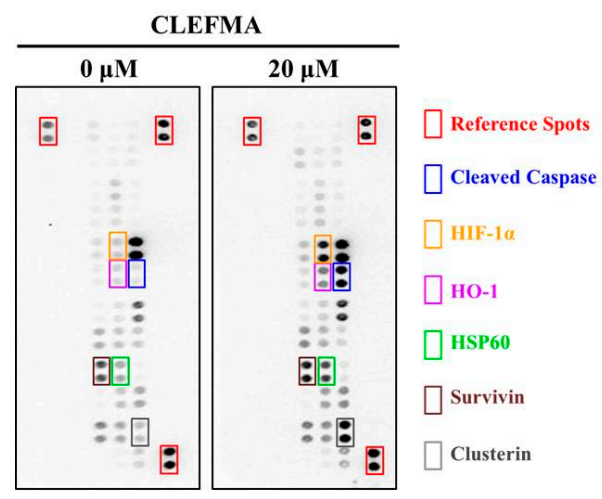

(B)

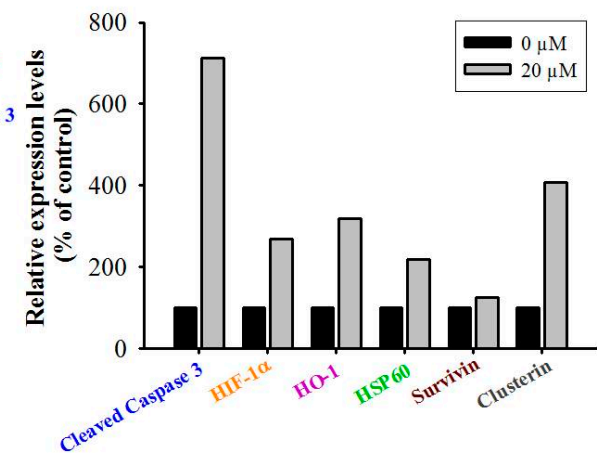

Figure 3. Effects of CLEFMA on the human apoptosis array in U2OS cells. (A) After treatment with $20 \mu \mathrm{M}$ CLEFMA for $24 \mathrm{~h}$ in U2OS cells, the human apoptosis array, with 35 apoptosis-related proteins included, was employed as described in the Materials and Methods. (B) The five increased proteins were subjected to quantitative analysis.

\subsection{CLEFMA Triggers Activation of the Caspase Cascade in U2OS Cells}

To investigate the effect of CLEFMA on the caspase cascade in the apoptotic signaling pathway, the effector caspase 3 and its upstream initiators, caspases 8 and 9, as well as their cleaved forms 
were determined with Western blotting. After treatment with different concentrations of CLEFMA in $\mathrm{U} 2 \mathrm{OS}$ cells for $24 \mathrm{~h}$, the higher concentrations of CLEFMA corresponded to higher expressions of the cleaved forms of caspases 3, 8, and 9, in a dose-dependent manner $(p<0.001, p<0.001$ and $p<0.001$, respectively), combined with the lesser expressions of caspases 3, 8, and 9, dose-dependently $(p<0.001$, $p<0.001$ and $p<0.001$, respectively). (Figure 4A-C) Thus, we found that CLEFMA induces U2OS cell apoptosis by activating both extrinsic caspase 8- and intrinsic caspase 9-mediated pathways and their downstream effector caspase 3 .
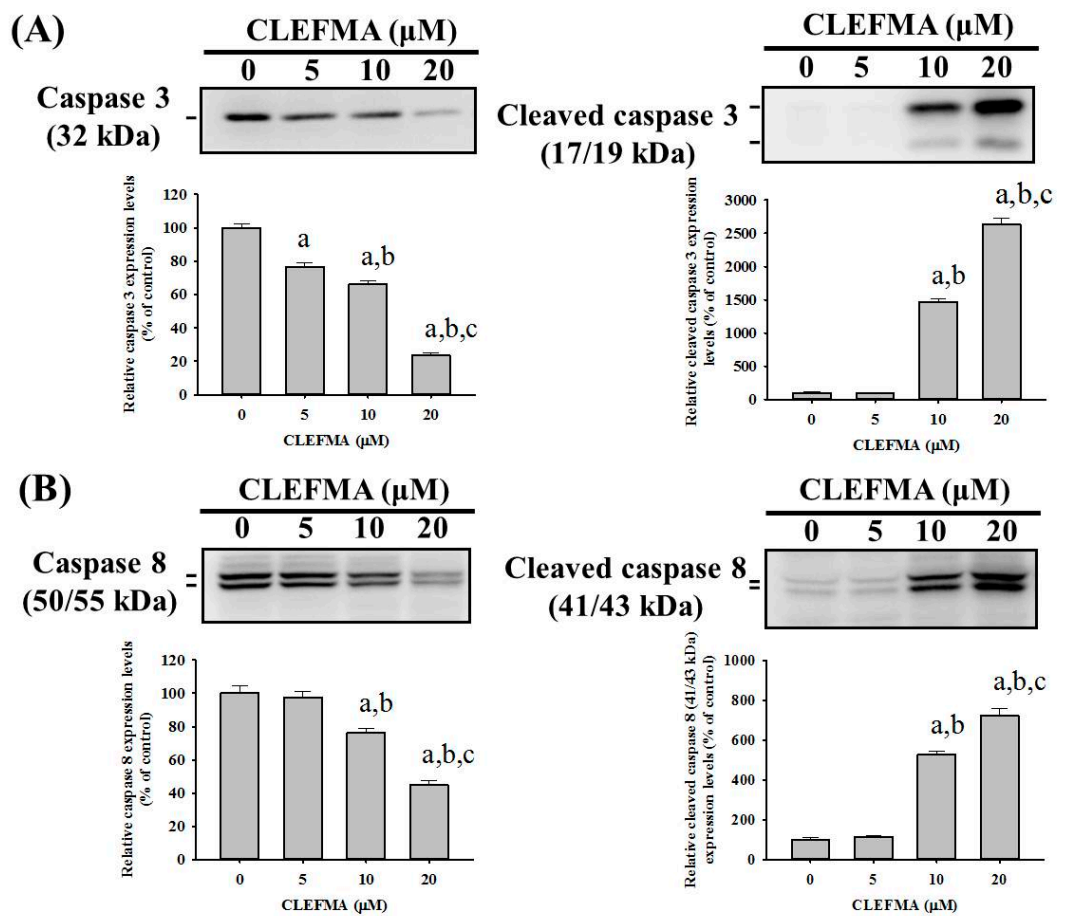

(C)
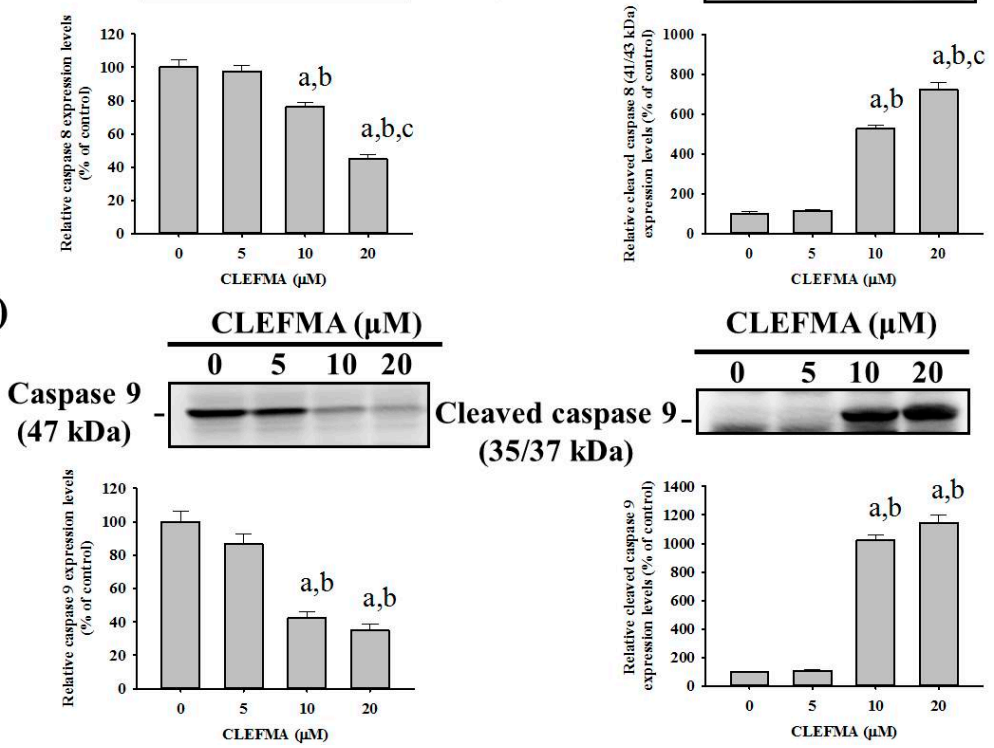

Figure 4. Effects of CLEFMA on the activation of caspases 3, 8 and 9 in U2OS cells. Western blot analysis for caspases 3,8 and 9 and their active forms after various concentrations $(5,10$ and $20 \mu \mathrm{M})$ of CLEFMA treatment for $24 \mathrm{~h}$ in U2OS cells were measured as described in the Materials and Methods. Subsequently, (A) caspase 3 and cleaved caspase 3, (B) caspase 8 and cleaved caspase 8 , and (C) caspase 9 and cleaved caspase 9 were subjected to quantitative analysis. Results are shown as mean \pm S.D.; $\mathrm{n}=3$. ANOVA analysis with Turkey's posteriori comparison was used. Caspase 3: $\mathrm{F}=196.205, p<0.001$; cleaved caspase 3: $\mathrm{F}=478.594, p<0.001$. Caspase 8: $\mathrm{F}=51.604, p<0.001$; cleaved caspase 8: $\mathrm{F}=205.373$, $p<0.001$. Caspase 9: $\mathrm{F}=37.754, p<0.001$; cleaved caspase 9: $\mathrm{F}=294.964, p<0.001$. a: Significantly different, $p<0.05$, when compared to control. B: Significantly different, $p<0.05$, when compared to $5 \mu \mathrm{M}$. c: Significantly different, $p<0.05$, when compared to $10 \mu \mathrm{M}$. 


\subsection{CLEFMA Activates Extrinsic and Intrinsic Apoptotic Processes via JNK and p38 Pathways in U2OS Cells}

Since MAPK pathways have been implicated as playing an important role in the action of chemotherapeutic drugs in the regulation of apoptosis and may be part of the signaling pathways that directly affect caspases 3,8, and 9, the Western blot analysis was employed to further investigate the underlying molecular mechanisms. As shown in Figure 5A-C, CLEFMA increased the phosphorylation of ERK1/2, JNK1/2 and p38, dose-dependently, in U2OS cells $(p<0.001, p<0.001$ and $p<0.001$, respectively), indicating that CLEFMA activates the phosphorylation of ERK1/2, JNK1/2 and p38 in U2OS cells. Furthermore, to identify whether the activation of ERK1/2, JNK1/2 and p38 phosphorylation by CLEFMA interferes with the actions of caspases 3, 8, and 9 of the extrinsic and intrinsic apoptotic processes in U2OS cells, we used inhibitors of ERK1/2 (U0126), JNK1/2 (JNK-in-8), and p38 (SB203580) with or without treatment with $20 \mu \mathrm{M}$ CLEFMA in Western blotting. Cleaved caspases 3, 8, and 9 were activated by $20 \mu \mathrm{M}$ of CLEFMA $(p<0.001, p<0.001$ and $p=0.001$ ), as expected. (Figure 6) Intriguingly, inhibitors of JNK1/2 (JNK-in-8) and p38 (SB203580) significantly repressed CLEFMA's increase of cleaved caspases 3, 8 and 9 in U2OS cells (JNK-in-8: $p<0.001, p<0.001$ and $p=0.013$; SB203580: $p<0.001, p<0.001$ and $p=0.003$ ), but the inhibitor of ERK1/2 (U0126) did not suppress CLEFMA's increase of cleaved caspases 3, 8 and 9 (U0126: $p=0.088, p=0.568$ and $p=0.990$ ). Overall, these findings indicated that JNK1/2 and p38 pathways play a critical upstream role in CLEFMA-mediated apoptosis of extrinsic caspase 8- and intrinsic caspase 9-mediated pathways and their downstream effector caspase 3 in U2OS cells.
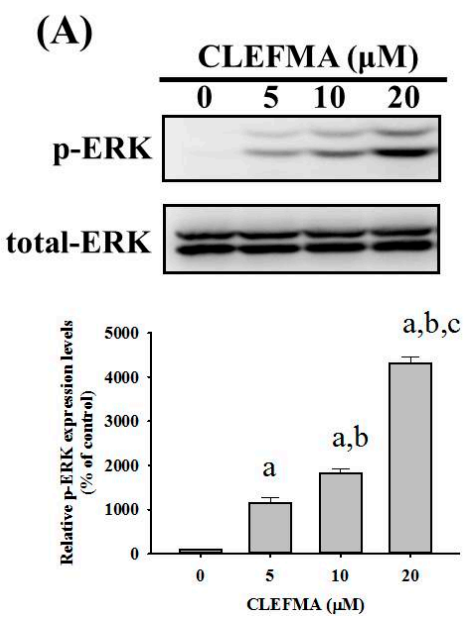

(B)
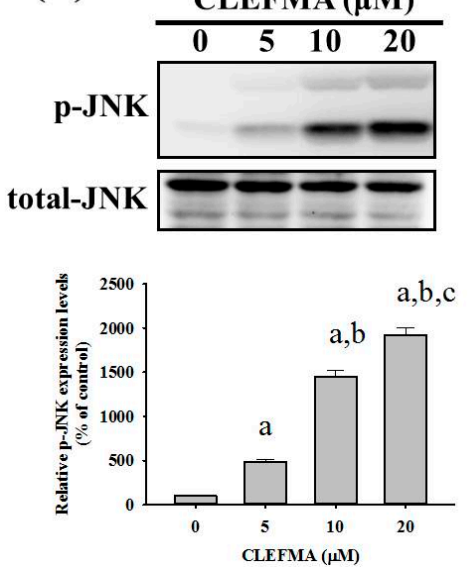
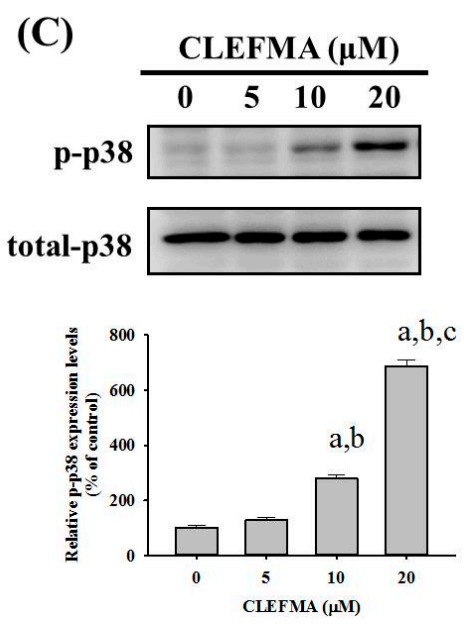

Figure 5. Effects of CLEFMA on the phosphorylation of ERK, c-Jun N-terminal kinases (JNK) and p38 in U2OS cells. Expressions of ERK1/2, JNK 1/2 and p38, as well as their phosphorylation after various concentrations $(5,10$ and $20 \mu \mathrm{M})$ of CLEFMA treatment for $24 \mathrm{~h}$ in U2OS cells, were measured through Western blot analysis. Next, they were subjected to quantitative analysis. Results are shown as mean \pm S.D.; $n=3$. ANOVA analysis with Turkey's posteriori comparison was used. (A) $p$-ERK: $\mathrm{F}=275.513, p<0.001 ;(\mathrm{B}) \mathrm{p}-\mathrm{JNK}: \mathrm{F}=205.474, p<0.001$; and $(\mathrm{C}) \mathrm{p}=\mathrm{p} 38: \mathrm{F}=292.128, p<0.001$. a: Significantly different, $p<0.05$, when compared to control. B: Significantly different, $p<0.05$, when compared to $5 \mu \mathrm{M}$. c: Significantly different, $p<0.05$, when compared to $10 \mu \mathrm{M}$. 

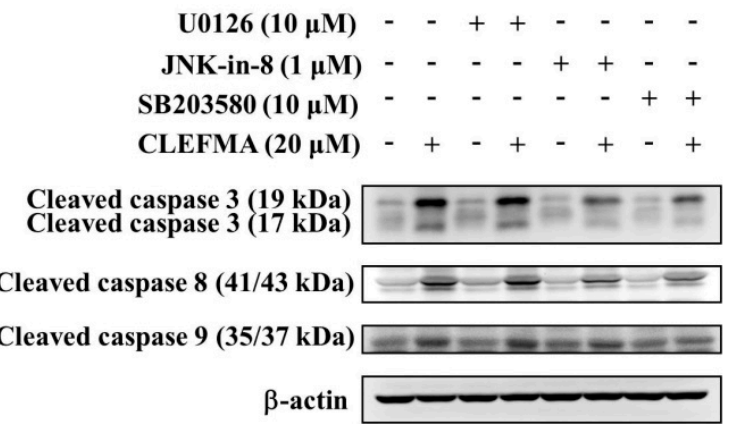

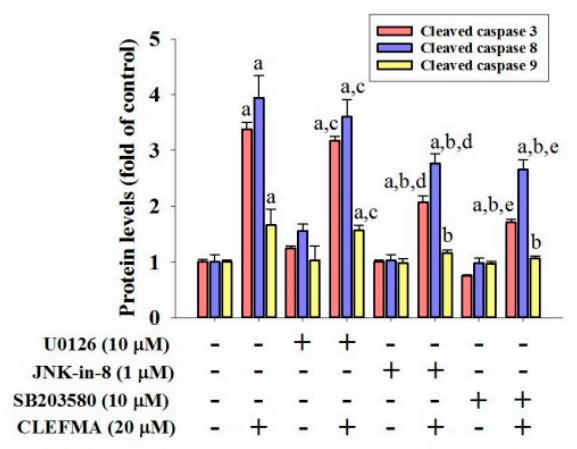

Figure 6. Effects of CLEFMA and inhibitors of ERK1/2 (U0126), JNK1/2 (JNK-in-8), and p38 (SB203580) on cleaved caspases 3, 8 and 9 expression of U2OS cells. Expressions of cleaved caspases 3, 8 and 9 after pretreatment with or without $10 \mu \mathrm{M}$ of U0126, $1 \mu \mathrm{M}$ of JNK-in-8, and $10 \mu \mathrm{M}$ of SB203580 for $1 \mathrm{~h}$ followed by $20 \mu \mathrm{M}$ or without CLEFMA treatment for an additional $24 \mathrm{~h}$ in U2OS cells were measured through Western blot analysis. Next, they were subjected to quantitative analysis. Results are shown as mean \pm S.D.; $n=3$. ANOVA analysis with Turkey's posteriori comparison was used. Cleaved caspase 3: $\mathrm{F}=502.398, p<0.001$; Cleaved caspase 8: $\mathrm{F}=95.967, p<0.001$; and cleaved caspase 9: $\mathrm{F}=10.543$, $p<0.001$. a: Significantly different, $p<0.05$, when compared to control. b: Significantly different, $p<0.05$, when compared to $20 \mu$ M CLEFMA. c: Significantly different, $p<0.05$, when compared to U0126. d: Significantly different, $p<0.05$, when compared to JNK-in-8. e: Significantly different, $p<0.05$, when compared to SB203580.

\section{Discussion}

In previous studies, curcumin has been reported to induce the apoptosis of human leukemia THP-1 cells through the activation of JNK/ERK pathways [21] and SHI-1 cells, possibly via both intrinsic and extrinsic pathways triggered by MAPKs (ERK, JNK and p38) signaling [22]. Also, curcumin exerts antitumor effects in retinoblastoma cells by regulating the JNK and p38 pathways [23], while this occurs through ERK1/2 and p38 signaling in malignant mesothelioma cells [24]. In human osteoclastoma cells, curcumin inhibits cell proliferation and promotes apoptosis through JNK, NF- $\mathrm{kB}$ and MMP-9 signaling pathways [25]. In spite of its efficacy and safety, curcumin has severely limited bioavailability because of its poor absorption and rapid metabolism [16].

After using the adjuvant to improve the poor bioavailability of curcumin, natural borneol and curcumin synergistically induce the apoptosis of human melanoma A375 cells with the involvement of the downregulation of Akt and ERK1/2 phosphorylation and the upregulation of phosphorylated JNK [26]. Similarly, the JNK/Bcl-2/Beclin1 pathway is thought to play a key role in the induction of apoptosis and autophagic cell death in breast cancer cells by the co-treatment of curcumin and berberine [27]. Additionally, synergistic inhibitory effects of cetuximab and curcumin on human cisplatin-resistant oral cancer CAR cells have been observed through the MAPK pathway and the intrinsic apoptotic process [28]. Moreover, curcumin-based photodynamic therapy induces breast cancer apoptosis through the activation of the ROS-mediated JNK/caspase-3 signaling pathway [29].

In managing patients diagnosed with any form of osteosarcoma, powerful chemotherapeutic drugs are the mainstay. Apart from adjuvants, structural analogues of curcumin (e.g., EF-24 and CLEFMA) have been undertaken to improve the bioavailability of curcumin for chemotherapy [16]. Although the synthetic curcuminoid CLEFMA developed over the past years has focused on anticancer effects against lung cancer cells $[17,18,20]$, no research has been reported on the apoptotic process of CLEFMA in osteosarcoma cells. Here, we intriguingly found that CLEFMA decreases cell viabilities and induces cell apoptosis in human osteosarcoma U2OS and HOS cells.

Currently, the process of apoptosis is triggered by two different signaling pathways. The extrinsic apoptotic signal, which responded mainly to extracellular stimuli, involves death receptors, and the intrinsic apoptotic process, activated by modulators within the cell itself, involves the mitochondria $[30,31]$. The action of the cascade of caspases is required to conduct apoptosis signal 
transduction and execution. As in other reports, we discovered that effector caspase 3 plays a critical role in the underlying programs of apoptosis and relies on the activation of its upstream initiators including extrinsic caspase 8 and intrinsic caspase 9 [8,32].

By collecting information from various aspects of signal transduction cascades and cellular metabolism, both pathways continuously process this signaling, and eventually decide on the fate of cells. While CLEFMA's phosphorylation of ERK1/2, JNK1/2 and p38 in U2OS cells was observed in the study, we supposed that CLEFMA's induction of the extrinsic and intrinsic apoptotic pathways was achieved through these three MAPK pathways. Unexpectedly, CLEFMA's increases of cleaved caspases 3,8 , and 9 could be effectively inhibited by co-treatment with inhibitors of JNK (JNK-in-8) and p38 (SB203580), but co-treatment with the ERK inhibitor (U0126) had no effect on the increased effect. Therefore, these findings suggested that CLEFMA activates both extrinsic and intrinsic apoptotic pathways in U2OS cells through JNK and p38 signaling, but the ERK pathway is not involved. CLEFMA's increases of cleaved caspases 3,8 , and 9 could be effectively inhibited with the co-treatment of the ERK inhibitor (U0126), implying that the cleaved caspases 3, 8, and 9 are not the downstream of the CLEFMA's phosphorylation of ERK1/2.

\section{Materials and Methods}

\subsection{Materials}

Cell culture materials including Dulbecco's modified Eagle medium (DMEM) and fetal bovine serum (FBS) were purchased from Gibco-BRL (Gaithersburg, MD, USA) and Hyclone Laboratories, Inc. (Logan, UT, USA), respectively. Antibodies specific for $\mathrm{p} 38$, phosphorylated $\mathrm{p} 38, \beta$-actin, caspases 3 and 8, and FITC (fluorescein isothiocyanate-labeled) Annexin V Apoptosis Detection Kit I were obtained from BD Biosciences (San Jose, CA, USA). Human Apoptosis Array Kit was purchased from R\&D Systems (Minneapolis, MN, USA). Additionally, antibodies specific for ERK1/2, JNK1/2, phosphorylated ERK1/2 and JNK1/2, caspases 9, and cleaved caspases 3, 8 and 9 were purchased from Cell Signaling Technology (Danvers, MA, USA). Unless otherwise specified, all chemicals used in this study were purchased from Sigma-Aldrich (St. Louis, MO, USA).

\subsection{Cell Culture and CLEFMA Treatment}

Obtained from the Food Industry Research and Development Institute (Hsinchu, Taiwan), the human osteosarcoma U2OS (15-year-old female) cells and HOS (13 year-old female) cells were supplemented with $10 \% \mathrm{FBS}, 1 \%$ penicillin/streptomycin, and $5 \mathrm{~mL}$ glutamine while being cultured in DMEM and Eagle's MEM, respectively. The cell cultures were maintained at $37^{\circ} \mathrm{C}$ in a humidified atmosphere of a $5 \%$ CO2 incubator. CLEFMA was purchased from Sigma-Aldrich (St. Louis, MO, USA).

\subsection{Microculture Tetrazolium Colorimetric (MTT) Assay}

To obtain information regarding the effect of apoptosis induced by CLEFMA, we subjected $8.5 \times 10^{4} /$ well U2OS cells and $7.5 \times 10^{4} /$ well HOS cells in 24-well plates for $16 \mathrm{~h}$ and treated them with different concentrations $(5,10,20,40$ and $80 \mu \mathrm{M})$ of CLEFMA to assay cell viability via MTT [3-(4,5-dimethylthiazol-2-yl)-2,5-diphenyltetrazolium bromide] assay. After the $24 \mathrm{~h}$ exposure period, the media were removed and the U2OS and HOS cells were washed with phosphate-buffered saline. Afterwards, the medium was changed and the cells were incubated with MTT $(0.5 \mathrm{mg} / \mathrm{mL})$ for $4 \mathrm{~h}[33,34]$.

\subsection{Annexin V-FITC Apoptosis Staining Assay}

About $8.5 \times 10^{5} \mathrm{U} 2 \mathrm{OS}$ and HOS cells in one $6 \mathrm{~cm}$ plate were cultured and treated with different concentrations $(0,5,10$ and $20 \mu \mathrm{M})$ of CLEFMA for $24 \mathrm{~h}$. Subsequently, U2OS cells were harvested with trypsinization together with floating non-viable cells. The FITC Annexin V Apoptosis Detection Kit I was used according to the manufacturer's protocols (BD Biosciences, San Jose, CA, USA); thereafter, 
the cell cycle analysis was measured by flow cytometry. Combined with PI staining, annexin V-FITC apoptosis staining was performed to differentiate apoptosis from necrosis.

\subsection{Human Apoptosis Array}

To explore the underlying mechanism of induced apoptosis, a Human Apoptosis Array Kit was used to evaluate protein lysates from vehicle- or $20 \mu \mathrm{M}$ CLEFMA-treated cells for $24 \mathrm{~h}$ according to the manufacturer's protocols (R\&D Systems, Minneapolis, MN). The kit detected 35 human apoptosis-related proteins simultaneously. Captured proteins were presented on the nitrocellulose membrane, detected with biotinylated detection antibodies, then finally visualized using chemiluminescent detection reagents.

\subsection{Protein Extraction and Western Blot Analysis}

To investigate the molecular mechanism further, the initiator and effector caspases and signaling pathways were detected using Western blot analysis. We plated $8.5 \times 10^{5} \mathrm{U} 2 \mathrm{OS}$ cells in $6 \mathrm{~cm}$ plates for $16 \mathrm{~h}$ and treated them with different concentrations $(0,5,10$ and $20 \mu \mathrm{M})$ of CLEFMA for $24 \mathrm{~h}$, and the total cell lysates of U2OS cells were prepared as described previously [33-35]. Western blot analysis was performed using specific primary antibodies against caspases 3, 8 and 9, cleaved caspases 3,8 and 9, and the specific antibodies for unphosphorylated or phosphorylated forms of the three corresponding MAPKs (ERK1/2, JNK1/2, and p38). As described previously, blots were then incubated with a horseradish peroxidase goat anti-rabbit or anti-mouse IgG for $1 \mathrm{~h}$, and the intensity of each band was measured via densitometry [33-35].

\subsection{Statistical Analysis}

Statistical calculations of the data were performed using one-way analysis of variance (ANOVA) with post hoc Scheffe's and Turkey's tests for more than two groups with unequal and equal sample sizes per group, respectively. Each experiment was performed in triplicate, and three independent experiments were performed. Statistical significance was at $p<0.05$.

\section{Conclusions}

Overall, these results demonstrated that CLEFMA decreases cell viabilities and induces the apoptosis of human osteosarcoma U2OS and HOS cells. By activating JNK and p38 pathways, but not via the ERK, both the extrinsic and intrinsic caspase cascades are triggered to induce the apoptosis of U2OS cells. Thus, CLEFMA may be a potential therapeutic agent against human osteosarcoma, whereas the therapeutic potential of CLEFMA combined with chemotherapy in osteosarcoma treatment should warrant evaluation in future research. Further tests are needed to investigate the detailed effects and possible mechanism of CLEFMA on the cell cycle progression and regulatory molecules of human osteosarcoma cells; however, animal studies are needed to justify CLEFMA as a promising candidate as a cytotoxic agent against osteosarcoma in vivo.

Author Contributions: Conceptualization, J.-S.Y., S.-F.Y. and K.-H.L.; methodology, R.-C.L., Y.-H.H., H.-H.W., G.-C.L., and Y.-C.L.; validation, J.-S.Y., S.-F.Y. and K.-H.L.; resources, S.-F.Y.; writing-original draft preparation, J.-S.Y., S.-F.Y. and K.-H.L.; writing—review and editing, J.-S.Y., S.-F.Y. and K.-H.L.

Funding: This research was funded by Chung Shan Medical University Hospital, Taiwan, grant number CSH-2017-D-003. This research was also funded by China Medical University, Taiwan (CMU 106-N-013).

Acknowledgments: The authors would like to express sincere thanks to Eric Wun-Hao Lu of American School in Taichung for proofreading.

Conflicts of Interest: The authors declare no conflict of interest. 


\section{References}

1. Mirabello, L.; Troisi, R.J.; Savage, S.A. Osteosarcoma incidence and survival rates from 1973 to 2004: Data from the surveillance, epidemiology, and end results program. Cancer 2009, 115, 1531-1543. [CrossRef] [PubMed]

2. Ottaviani, G.; Jaffe, N. The epidemiology of osteosarcoma. Cancer Treat. Res. 2009, 152, 3-13. [PubMed]

3. Oertel, S.; Blattmann, C.; Rieken, S.; Jensen, A.; Combs, S.E.; Huber, P.E.; Bischof, M.; Kulozik, A.; Debus, J.; Schulz-Ertner, D. Radiotherapy in the treatment of primary osteosarcoma-A single center experience. Tumori 2010, 96, 582-588. [CrossRef]

4. Hengartner, M.O. The biochemistry of apoptosis. Nature 2000, 407, 770-776. [CrossRef]

5. Davis, R.J. Signal transduction by the jnk group of map kinases. Cell 2000, 103, 239-252. [CrossRef]

6. Karin, M.; Cao, Y.; Greten, F.R.; Li, Z.W. Nf-kappab in cancer: From innocent bystander to major culprit. Nat. Rev. Cancer 2002, 2, 301-310. [CrossRef]

7. Fulda, S.; Debatin, K.M. Targeting apoptosis pathways in cancer therapy. Curr. Cancer Drug Targets 2004, 4, 569-576. [CrossRef] [PubMed]

8. Lu, K.H.; Chen, P.N.; Lue, K.H.; Lai, M.T.; Lin, M.S.; Hsieh, Y.S.; Chu, S.C. 2'-hydroxyflavanone induces apoptosis of human osteosarcoma $143 \mathrm{~b}$ cells by activating the extrinsic trail- and intrinsic mitochondria-mediated pathways. Nutr. Cancer 2014, 66, 625-635. [CrossRef] [PubMed]

9. Degterev, A.; Boyce, M.; Yuan, J. A decade of caspases. Oncogene 2003, 22, 8543-8567. [CrossRef] [PubMed]

10. Kunnumakkara, A.B.; Bordoloi, D.; Harsha, C.; Banik, K.; Gupta, S.C.; Aggarwal, B.B. Curcumin mediates anticancer effects by modulating multiple cell signaling pathways. Clin. Sci. (Lond.) 2017, 131, 1781-1799. [CrossRef]

11. Chang, Z.; Xing, J.; Yu, X. Curcumin induces osteosarcoma mg63 cells apoptosis via ros/cyto-c/caspase-3 pathway. Tumour. Biol. 2014, 35, 753-758. [CrossRef] [PubMed]

12. Jin, S.; Xu, H.G.; Shen, J.N.; Chen, X.W.; Wang, H.; Zhou, J.G. Apoptotic effects of curcumin on human osteosarcoma u2os cells. Orthop. Surg. 2009, 1, 144-152. [CrossRef] [PubMed]

13. Lee, D.S.; Lee, M.K.; Kim, J.H. Curcumin induces cell cycle arrest and apoptosis in human osteosarcoma (hos) cells. Anticancer Res. 2009, 29, 5039-5044. [PubMed]

14. Singh, M.; Pandey, A.; Karikari, C.A.; Singh, G.; Rakheja, D. Cell cycle inhibition and apoptosis induced by curcumin in ewing sarcoma cell line sk-nep-1. Med. Oncol. 2010, 27, 1096-1101. [CrossRef] [PubMed]

15. Walters, D.K.; Muff, R.; Langsam, B.; Born, W.; Fuchs, B. Cytotoxic effects of curcumin on osteosarcoma cell lines. Invest. New Drugs 2008, 26, 289-297. [CrossRef]

16. Anand, P.; Kunnumakkara, A.B.; Newman, R.A.; Aggarwal, B.B. Bioavailability of curcumin: Problems and promises. Mol. Pharm. 2007, 4, 807-818. [CrossRef] [PubMed]

17. Lagisetty, P.; Vilekar, P.; Sahoo, K.; Anant, S.; Awasthi, V. Clefma-an anti-proliferative curcuminoid from structure-activity relationship studies on 3,5-bis(benzylidene)-4-piperidones. Bioorg. Med. Chem. 2010, 18, 6109-6120. [CrossRef]

18. Sahoo, K.; Dozmorov, M.G.; Anant, S.; Awasthi, V. The curcuminoid clefma selectively induces cell death in h441 lung adenocarcinoma cells via oxidative stress. Investig. New Drugs 2012, 30, 558-567. [CrossRef]

19. Raghuvanshi, D.; Nkepang, G.; Hussain, A.; Yari, H.; Awasthi, V. Stability study on an anti-cancer drug 4-(3,5-bis(2-chlorobenzylidene)-4-oxo-piperidine-1-yl)-4-oxo-2-butenoic acid (clefma) using a stability-indicating hplc method. J. Pharm. Anal. 2017, 7, 1-9. [CrossRef]

20. Yadav, V.R.; Sahoo, K.; Awasthi, V. Preclinical evaluation of 4-[3,5-bis(2-chlorobenzylidene)-4-oxo-piperidine1-yl]-4-oxo-2-butenoic acid, in a mouse model of lung cancer xenograft. Br. J. Pharmacol. 2013, 170, 1436-1448. [CrossRef]

21. Yang, C.W.; Chang, C.L.; Lee, H.C.; Chi, C.W.; Pan, J.P.; Yang, W.C. Curcumin induces the apoptosis of human monocytic leukemia thp-1 cells via the activation of jnk/erk pathways. BMC Complement. Altern. Med. 2012, 12, 22. [CrossRef] [PubMed]

22. Zhu, G.H.; Dai, H.P.; Shen, Q.; Ji, O.; Zhang, Q.; Zhai, Y.L. Curcumin induces apoptosis and suppresses invasion through mapk and mmp signaling in human monocytic leukemia shi-1 cells. Pharm. Biol. 2016, 54, 1303-1311. [CrossRef] [PubMed]

23. Yu, X.; Zhong, J.; Yan, L.; Li, J.; Wang, H.; Wen, Y.; Zhao, Y. Curcumin exerts antitumor effects in retinoblastoma cells by regulating the jnk and p38 mapk pathways. Int. J. Mol. Med. 2016, 38, 861-868. [CrossRef] [PubMed] 
24. Masuelli, L.; Benvenuto, M.; Di Stefano, E.; Mattera, R.; Fantini, M.; De Feudis, G.; De Smaele, E.; Tresoldi, I.; Giganti, M.G.; Modesti, A.; et al. Curcumin blocks autophagy and activates apoptosis of malignant mesothelioma cell lines and increases the survival of mice intraperitoneally transplanted with a malignant mesothelioma cell line. Oncotarget 2017, 8, 34405-34422. [CrossRef] [PubMed]

25. Cao, F.; Liu, T.; Xu, Y.; Xu, D.; Feng, S. Curcumin inhibits cell proliferation and promotes apoptosis in human osteoclastoma cell through mmp-9, nf-kappab and jnk signaling pathways. Int. J. Clin. Exp. Pathol. 2015, 8, 6037-6045. [PubMed]

26. Chen, J.; Li, L.; Su, J.; Li, B.; Chen, T.; Wong, Y.S. Synergistic apoptosis-inducing effects on a375 human melanoma cells of natural borneol and curcumin. PLOS ONE 2014, 9, e101277. [CrossRef] [PubMed]

27. Wang, K.; Zhang, C.; Bao, J.; Jia, X.; Liang, Y.; Wang, X.; Chen, M.; Su, H.; Li, P.; Wan, J.B.; et al. Synergistic chemopreventive effects of curcumin and berberine on human breast cancer cells through induction of apoptosis and autophagic cell death. Sci. Rep. 2016, 6, 26064. [CrossRef] [PubMed]

28. Chen, C.F.; Lu, C.C.; Chiang, J.H.; Chiu, H.Y.; Yang, J.S.; Lee, C.Y.; Way, T.D.; Huang, H.J. Synergistic inhibitory effects of cetuximab and curcumin on human cisplatin-resistant oral cancer car cells through intrinsic apoptotic process. Oncol. Lett. 2018, 16, 6323-6330. [CrossRef]

29. Sun, M.; Zhang, Y.; He, Y.; Xiong, M.; Huang, H.; Pei, S.; Liao, J.; Wang, Y.; Shao, D. Green synthesis of carrier-free curcumin nanodrugs for light-activated breast cancer photodynamic therapy. Colloids Surf. B Biointerfaces 2019, 180, 313-318. [CrossRef]

30. Gazitt, Y.; Kolaparthi, V.; Moncada, K.; Thomas, C.; Freeman, J. Targeted therapy of human osteosarcoma with 17aag or rapamycin: Characterization of induced apoptosis and inhibition of mtor and akt/mapk/wnt pathways. Int. J. Oncol. 2009, 34, 551-561. [CrossRef]

31. Park, H.; Bergeron, E.; Senta, H.; Guillemette, K.; Beauvais, S.; Blouin, R.; Sirois, J.; Faucheux, N. Sanguinarine induces apoptosis of human osteosarcoma cells through the extrinsic and intrinsic pathways. Biochem. Biophys. Res. Commun. 2010, 399, 446-451. [CrossRef] [PubMed]

32. Fulda, S.; Debatin, K.M. Extrinsic versus intrinsic apoptosis pathways in anticancer chemotherapy. Oncogene 2006, 25, 4798-4811. [CrossRef] [PubMed]

33. Hsieh, Y.S.; Chu, S.C.; Yang, S.F.; Chen, P.N.; Liu, Y.C.; Lu, K.H. Silibinin suppresses human osteosarcoma mg-63 cell invasion by inhibiting the erk-dependent c-jun/ap-1 induction of mmp-2. Carcinogenesis 2007, 28, 977-987. [CrossRef] [PubMed]

34. Lu, K.H.; Yang, H.W.; Su, C.W.; Lue, K.H.; Yang, S.F.; Hsieh, Y.S. Phyllanthus urinaria suppresses human osteosarcoma cell invasion and migration by transcriptionally inhibiting u-pa via erk and akt signaling pathways. Food Chem. Toxicol. 2013, 52, 193-199. [CrossRef] [PubMed]

35. Lu, K.H.; Chen, P.N.; Hsieh, Y.H.; Lin, C.Y.; Cheng, F.Y.; Chiu, P.C.; Chu, S.C.; Hsieh, Y.S. 3-hydroxyflavone inhibits human osteosarcoma u2os and $143 \mathrm{~b}$ cells metastasis by affecting emt and repressing u-pa/mmp-2 via fak-src to mek/erk and rhoa/mlc2 pathways and reduces $143 \mathrm{~b}$ tumor growth in vivo. Food Chem. Toxicol. 2016, 97, 177-186. [CrossRef] [PubMed]

Sample Availability: Not available.

(C) 2019 by the authors. Licensee MDPI, Basel, Switzerland. This article is an open access article distributed under the terms and conditions of the Creative Commons Attribution (CC BY) license (http://creativecommons.org/licenses/by/4.0/). 